\title{
The Vicissitudes of Mathematical Reason in the 20th Century
}

Paolo Mancosu, The Adventure of Reason. Interplay Between Philosophy of Mathematics and Mathematical Logic, 1900 - 1940, Oxford University Press, New York, 2010, Hardback, \$81.52, 618 pages.

The nature of reason is a central theme of philosophy since its inception, and has provoked controversies ever since. In The Adventure of Reason (henceforth Adventure) Mancosu deals with a part of reason that, for the majority of time, has managed to stay outside most of the troubles that affected general reason during the course of history. More precisely, Adventure deals with reason restricted to the realms of mathematics, logic, and the sciences.

As Mancosu reveals in the preface, the book's title is borrowed from Oskar Becker (1889 - 1964), a now largely forgotten German phenomenological philosopher of science and mathematics. Becker, who had been a student of Husserl and Heidegger, took the concept of an „adventurous reason" from Goethe, who used it to distinguish his „intuitive science“, in which 'intuition' played a pre-eminent role, from the Newtonian science favored by Kant and other philosophers of the enlightenment. For Becker "the adventure of reason" was more than just a "beautiful image" (as it seems to be for Mancosu). As this review will describe, 'intuition' in a vaguely Goethian sense played an important role in the evolution of reason in the early decades of the 20th century, among many philosophers and scientists belonging to the German Kulturkreis.

In order to address the vicissitudes of mathematical reason in the first half of the 20th century Mancosu concentrates on the interplay between philosophy of mathematics and mathematical logic that shaped the contemporary conceptual landscape to which logic, mathematics, the sciences, and philosophy belong. Adventure has five parts:

I. History of logic (with Richard Zach and Calixto Badesa)

II. Foundation of Mathematics

III. Phenomenology and the Exact Sciences (with Thomas Ryckman)

IV. Quine and Tarski on Nominalism

V. Tarski and the Vienna Circle on Truth and Logical Consequence 
Adventure is a compilation of texts all of which have been published elsewhere in the last ten years or so. They greatly vary in style and character: some chapters offer authoritative surveys, others may be characterized as miscellanea dealing with details of the history of mathematics, while still others intend to contribute to contemporary philosophical debates concerning logic and epistemology. Nevertheless, Mancosu contends, "the essays are closely linked by the fact that the subject matter is homogeneous and were written with a single major aim, namely that of reaching a deeper understanding of the interaction between developments in mathematical logic and the foundations of mathematics and logic from 1900 -1940." The reader may decide for himself, whether he accepts this argument as convincing or not.

Part I (Chapter 1) History of Logic, written jointly with Richard Zach and Calixto Badesa, offers a fairly standard account of the development of mathematical logic in the first third of the 20th century. Without too much historiographical baggage and philosophical interpretation the authors offer eight "itineraries" starting with a succinct discussion of the basic meta-axiomatic concepts such as consistency, completeness, categoricity and so on, as they were developed by members of the Italian school around the turn of the century. Then, a brief overview of Russell's mathematical logic is described, beginning with Principles of Mathematics and progressing to the Principia Mathematica. Next follows Zermelo's formalization of set theory and the beginnings of model theory in Löwenheim's Theory of Relatives. The subsequent sections are dedicated to the logic of the Hilbert school (Hilbert, Bernays, Ackermann, Gentzen, and others). As is well known Hilbert's program of the logical foundations of mathematics failed in the light of Gödel's incompleteness theorems. These are also addressed in Part I, albeit briefly.

Another itinerary then concentrates on the topics of intuititionism and explores manyvalued logics beginning with Brouwer and ultimately progressing to Gödel's clarification of the relation between classical and intuititionist logic, asserting that all arithmetic propositions provable from the classical axioms can be translated into ones that are theorems of intuitionist logic. The chapter concludes with a presentation of some early results of Huntington, Post and others concerning what, in modern terms, may be called model theory, culminating in Tarski's theory of truth. Some readers may be disappointed that there is no 'American' itinerary that addresses the contributions of the American pragmatists Peirce and C.I. Lewis.

Part II (Chapter 2 - Chapter 8) Foundations of Mathematics on the foundations of mathematics consists of seven short chapters that are predominately concerned with 
history of mathematics. One group, Chapter 2 - Chapter 4 , deals with the evolution of Hilbert's metamathematical programme in the decade between 1910 and 1920. This sections place particular focus on the growing influence of Russell and Whitehead's Principia Mathematica in Göttingen. Mancosu points out that one of the reasons for this development was the work of the logician and philosopher Heinrich Behmann. In 1914 Behmann gave a lecture on the Principia, later he wrote a dissertation (under Hilbert) that made intense use of some of Russell and Whitehead's results. The second group of miscellanea contained within Part II, Chapter 5 and Chapter 6, deals with questions concerning the constructivity of mathematical proofs. Chapter 5 addresses a conjecture of Felix Kaufmann according to which all proofs of classical mathematics, which do not use the axiom of choice, were already "constructive". Behmann claimed to have a proof, but eventually Gödel provided an elementary counter-example. Chapter 6 (written jointly with Mathieu Marion) discusses Wittgenstein's efforts to render constructive a (nonconstructive) proof of Euler of the existence of infinitely many primes. The collection of pieces of this part is completed by a short report that describes the reception of Gödel's incompleteness theorems immediately after their announcement at the conference in Königsberg in September 1930. It is concludes with review of the edition of the last two volumes IV and V of Gödel's Collected Works.

PART III (Chapter 9 - Chapter 12) Phenomenology and the Exact Sciences, written partly in collaboration with Thomas Ryckman, could have been philosophically the most interesting piece of Adventure. For reasons to be explained in the following, it suffers from some shortcomings, however.

In Part III the authors go beyond the boundaries of the standard Anglo-Saxon analytic philosophy by treating Husserlian phenomenology as an important source for philosophy of science and mathematics in 20th century. For many contemporary analytic philosophers, phenomenology and the sciences belong to different intellectual worlds. This starkly contrasts with the assessment of the founding generation of the phenomenological movement. For instance, Husserl contended that transcendental phenomenology provided the only truly scientific foundation of mathematics and the exact sciences. The alleged affinity between phenomenology and the sciences was more than wishful thinking from the side of philosophers. A significant number of eminent logicians, mathematicians, and scientists, e.g. Gödel, Heyting, Weyl, and Fritz London (cf. Gavroglu 1995), took a version of phenomenology serious during at least at one point of their careers and many of them considered it as an important philosophical inspiration for their work. However, the 
majority of analytic philosophers dismissed claims that phenomenology could be a scientific philosophy and many of them ignored phenomenology altogether. Hence Mancosu's and Ryckman's attempts to overcome this state of affairs will be warmly welcomed by many readers.

As the main witness for the relevance of phenomenology for science and philosophy of science Hermann Weyl is called in. Chapter 9 is dedicated to a discussion of Weyl's contributions to the Hilbert and Brouwer debate on the foundations of mathematics that took place in the 1920s. Mancosu distinguishes four different phases. The first is characterized by Weyl's attempt of rendering precise the vague idea of a "definite property“, which played an important role in Zermelo's axiomatization of set theory. The second phase concentrates on Weyl's position put forward in Das Kontinuum. There he directly attacked set theory and emphasized the essential difference between the setthoretically constructed real numbers and the intuitive continuum of inner temporal experiences. When, a few years later, he became acquainted with Brouwer's radically antiHilbertian intuitionism he moved closer to Brouwer's theories but eventually sought to find a constructivist middle position between Hilbert and Brouwer.

The extant correspondence between Weyl and the German phenomenologist and philosopher of mathematics Oskar Becker was published with detailed commentaries and interpretations some years ago in (Mancosu and Ryckman 2002, 2005). In Adventure these articles are republished as Chapters 10 and 11 - regrettably without the letters themselves. Chapter 12 deals with the correspondence between Oskar Becker and his fellow-phenomenologist Dietrich Mahnke (1884 - 1939). Its thirteen letters have been published in Peckhaus (2005) but, regrettably, are not included in Adventure.

A more serious shortcoming of this part of Adventure concerns the fact that the authors fail to adequately address the cultural and philosophical background of the protagonists largely in the dark. For instance, Becker's thesis that every culture had its own concepts of numbers is directly taken from Oswald Spengler's Decline of the West, Spengler being at that time one of the leading figures of "a politics of cultural despair" (Fritz Stern 1974) in Germany. Becker's claim that the applicability of mathematics is an "undeserved luck“ is far from being a personal opinion but rather an expression of a wide-spread irrationalist Zeitgeist. Indeed, Becker is a telling example for the very strange vicissitudes of reason of the past century: In the 1920s we find him discussing subtle issues of philosophy of geometry and science with Reichenbach and Weyl, some years later he put forward a „paraexistential philosophy“ as an 'improvement' of Heidegger's in that his 
„paraexistentialism“ provided a more adequate grounding for central concepts of National Socialism (cf. Sluga 1995, Hogrebe 2009). Mancosu and Ryckman don't deal with the influence of this Weimar culture. Worse still, they also present the phenomenological background of the protagonists only in a rudimentary way. This may render the reading of this part of Adventure difficult to those readers who are accostumed only to the analytical way of doing philosophy of science. For instance, it can hardly be assumed that, say, eidetic intuition (Wesenserschauung) is a household term among contemporary philosophers and logicians. Perhaps a remedy for this shortcoming, which would have fitted well the multiauthored character of some chapters of Adventure would have been a re-publication of Mahnke's succinct First Introduction to Phenomenology, especially that of Formal Mathematics (Mahnke 1923 (1977)). For a thorougher presentation of Weyl's phenomenological involvement the reader may wish to consult the recent book of Mancosu's co-author Ryckman (2005, Chapter 5 and 6).

Within Part IV (Chapter 13 and Chapter 14) Quine and Tarski on Nominalism we return to the mainstream of analytic philosophy and history of logic and science. The two chapters contained within this part aim to elucidate some of the nominalist episodes in the philosophical careers of Quine, Tarski, and Carnap, which took place when they met at Princeton in the 1940s. The protagonists conceived nominalism as sort of an intellectual experiment designed to find out how far one could pursue the program of elimination of the "unthingly“ without sacrificing science. This program was structured in two stages. The first consisted in identifying a nominalist system of mathematics; the second stage was to provide a reconstruction of science on that base. A few years later Quine (and Carnap) gave up their flirtation with nominalism, arriving at a more tolerant attitude with respect to ontological matters, as expressed in Empiricism, Semantics and Ontology (Carnap 1950). Mancosu points out the nominalism of Quine, Tarski, and Carnap was motivated mainly by their antipathy against an overly generous metaphysics - inherited from the doctrines of the Vienna Circle - and less by epistemological qualms related to Benacerraf's dilemma as is the case for contemporary nominalism.

PART V (Chapter 15 - Chapter 18) Tarski and the Vienna Circle on Truth and Logical Consequence deals with two central notions of 20th century logic and philosophy of science, namely, truth and logical consequence. Its protagonist is Tarski. Tarski's semantic conception of truth made its first public appearance on the International Congress for Unity of Science 1935 in Paris. His proposals found a mixed reception among the members of the Vienna Circle. While Carnap whole-heartedly welcomed Tarskian semantics, others, 
such as Neurath, suspected that the concept of truth would reintroduce "metaphysics" through the backdoor of formalization. The (unpublished) correspondence between Neurath and Carnap provides evidence that this was not a minor quarrel - the dispute about semantics almost led to the collapse of their friendship.

As Mancosu points out, albeit Tarski is rightly to be considered as the founding father of formal semantic it would be too simple to identify his concepts of truth and other basic semantic notion with those of modern semantics. Mancosu argues that Tarski's notion of logical consequence is at odds with the modern one, since it is characterized as a "fixed domain" conception of logical consequence. His argumentation is based on a step-by-step commentary on an hitherto unpublished lecture of Tarski's "On the Completeness and Categoricity of Deductive Systems" (1940) that is added as an appendix of Adventure.

Let us take stock. Mancosu's Adventure offers a rich panorama of ideas, theories, and arguments that have constituted the complex fabric of mathematical reason as it was woven by many great minds in the last century. It touches upon a bewildering manifold of issues. Nevertheless, in my opinion, Adventure ignores - or at least undervalues - an important aspect of mathematical reason, namely the one that connects it more closely with the world. In other words, the discussions of the problem of the applicability of mathematical knowledge to the world in Adventure leave something to desire. Even logicians and philosophers of mathematics with strong logicist inclinations such as Frege and Carnap required that a fully satisfying logicist foundation of mathematics had to explain the applicability of mathematics. A formal deduction of the axioms of mathematics from the laws of logic did not suffice. As Frege put it: "It is applicability alone which raises arithmetic above the game to the status of a science. Thus, applicability is an essential part." (Frege 1903, p. 100). Similarly, Carnap insisted on an integral philosophy of mathematics that should explain its applicability (cf. Syntax, 327). Whether they really lived up to this imperative in their philosophies, is, of course, a matter of dispute. In any case, an account of mathematics that conceived the applicability of mathematics as an „undeserved luck“ (Becker) would hardly satisfied them.

Carnap's and Frege's logicist projects are not alone in their emphasis on the applicability of mathematics for modern mathematical reason. They share it with an other important 20th century current of philosophy of science, logic and mathematics that regrettably is not treated at all in Adventure, to wit, the Neokantianism of the Marburg school. For instance, early in 20th century Ernst Cassirer contended in Kant und die moderne Mathematik: 
"If one is allowed to express the relation between philosophy and science in a blunt and paradoxical way, one may say: The eye of philosophy must be directed neither on mathematics nor on physics; it is to be directed solely on the connection of the two realms." (Cassirer 1907, 48)

For him, a philosophy of mathematics that conceived mathematical knowledge as an abstract edifice separated from applications in the exact sciences seriously misunderstood the role of mathematical reason in the context of general reason. Cassirer was seriously engaged in finding for a common root from which both physics and mathematics sprang he believed to have found it in the method of introducing ideal elements.

Cassirer and, more generally, the Neokantian contributions to philosophy of mathematics, logic and the sciences do not show up in Mancosu's narration of the adventures of (mathematical) Reason, and the philosophical problem of the applicability of mathematics receives only an insufficient treatment in his account. Despite of this and the somewhat meagre presentation of the phenomenological background of some of the protagonists and their general cultural background in Part III Adventure offers to the reader a rich and variegated panorama of some of the key episodes of the evolution of mathematical reason in the early $20 \mathrm{~h}$ century.

\section{References}

Cassirer, Ernst. 1907. Kant und die moderne Mathematik, Kant-Studien 12: 1- 49.

Feist, Robert. 2004. Husserl and the Sciences (ed.), Ottawa: University of Ottawa Press. Frege, Gottlob., 1903 (1962). Grundgesetze der Arithmetik, Darmstadt: Wissenschaftliche Buchgesellschaft.

Gavroglu, Kostas. 1995. Fritz London. A Scientific Biography, Cambridge: Cambridge University Press.

Hogrebe, Wolfgang. 2008. Die Selbstverstrickung des Philosophen Oskar Becker, in Hans Jörg Sandkühler (Hrg.), Philosophie im Nationalsozialismus, 157 - 190. Meiner: Hamburg. Mahnke, Dietrich. 1923 (1977). From Hilbert to Husserl: First Introduction to Phenomenology, Especially that of Formal Mathematics. Translated by D. Boyer. Studies in the History and Philosophy of Science 8: 71 - 84.

Mancosu, Paolo, Thomas Ryckman. 2002. The Correspondence between Hermann Weyl and Oskar Becker, Philosophia Mathematica 10: 130 - 202. 
Ryckman, Thomas. 2005. The Reign of Relativity: Philosophy of Physics 1915 - 1925. Oxford. Oxford University Press.

Sluga, Hans. 1994. Heidegger's Crisis, Philosophy and Politics in Nazi Germany, Cambridge/ Massachusetts. Harvard University Press.

Stern, Fritz. 1974. The Politics of Cultural Despair. A Study in the Rise of the Germanic Ideology, Berkeley, Los Angeles. The University of California Press.

Thomas Mormann, Department of Logic and Philosophy of Science, University of the Basque Country UPVEPU, Donostia-San Sebastian, Spain, ylxmomot@sf.ehu.es 